\title{
Energy Efficiency and Throughput Optimization of Cognitive Relay Networks
}

\author{
Yaolian Song, Fan Zhang and Shao Yubin \\ Faculty of Information Engineering and Automation, Kunming University of Science and Technology, Kunming, China
}

\begin{abstract}
In this paper, we investigate the energy efficiency and throughput optimization problem of cognitive relay networks. We propose to design sensing time and signal to noise ratio (SNR) to maximize the energy efficiency and throughput, since analytical and empirical studies have shown that sensing time and SNR are key factors for energy efficiency and throughput. We design a method that simultaneously considers the parameters of spectrum sensing time and SNR to optimize the energy efficiency of cognitive radio networks. Furthermore, we conduct deep experiments which show that there exists the optimal sensing time to maximize energy efficiency and throughput. In addition, optimal sensing time and optimal SNR can be jointly designed to maximize energy efficiency. Finally, we provide simulation results to show that energy efficiency of cognitive relay transmission scheme can be significantly improved compared with that of direct transmission scheme in cognitive radio networks.
\end{abstract}

Keywords: energy efficiency, cognitive relay transmission, SNR, spectrum sensing time

\section{Introduction}

Due to the fast development of wireless communication networks and mobile users, the demand for wireless radio spectrum is rapidly increasing. Cognitive radio network has received substantial attention as a promising approach to increasing spectrum efficiency. Cognitive radio enables opportunistic secondary spectrum access and allows secondary users to utilize the licensed frequency bands as long as the interference to the primary users is limited to an acceptable level [1]. Previous works on cognitive radio network focused on sensing accuracy, spectral efficiency and throughput [2-4].
Cognitive radio can improve spectral efficiency, meanwhile it will lead to lower energy efficiency due to external time overhead and energy consumption for spectrum sensing. Recent efforts toward techniques for energy efficiency and spectral efficiency have tended toward the concept of the energy efficient cognitive radio [5-8]. For instance, energy efficient sensing-access strategies for sequential sensing were addressed in [5], the energy-efficient transmission with variable transmission duration and interference limited to primary user(PU) were discussed when the sensing duration was fixed. Link adaptive transmission was addressed in [6] to maximize the energy efficiency. In [8], the authors jointly determined sensing duration and transmission duration for an energy-efficient design of cognitive radio networks by assuming that the PU does not reoccupy the channel during cognitive user transmission. Energyharvesting cognitive radio network can improve both energy efficiency and spectral efficiency with a secondary transmitter powered by renewable energy [10-12].

Inspired by the previous work and practical scenarios, in this paper we investigated the energy efficiency and the throughput of cognitive radio network in which cognitive user can periodically monitor the licensed frequency band to decide whether to transmit data or wait for the next frame. We establish an energy-efficient network model described by two variables, namely, sensing duration and signal noise ratio. Cognitive radio networks can enhance energy efficiency as well as spectral efficiency by simultaneously considering SNR and spectrum sensing 
duration. Energy detector and cognitive relay transmission scheme are addressed. We find that there exist optimal sensing duration and an optimal SNR to achieve the maximum of energy efficiency.

The paper is organized as follows: Section 2 depicts the system model. In Section 3, we show the throughput optimization and energy efficiency optimization of cognitive relay networks. In Section 4, numerical and simulation results are demonstrated and the paper is concluded in Section 5.

\section{System Model}

We consider cognitive relay model setup illustrated in Figure 1, which consists of a single cognitive source (CS), a single cognitive relay (CR) and a single cognitive destination (CD). PU denotes primary user. The cognitive relay operates in half-duplex AF mode, each transmission link between any two nodes is modeled as a Rayleigh fading channel.

The whole cognitive transmit process of cognitive system can be divided into three phases: spectrum sensing, cognitive source broadcasting information to cognitive relay and cognitive destination, cognitive relay forwarding information to cognitive destination in AF mode. Finally, the cognitive destination combines received information from cognitive source and cognitive relay in maximum-ratio combining. If cognitive user didn't detect idle licensed spectrum, all cognitive users would keep silent. Sup-

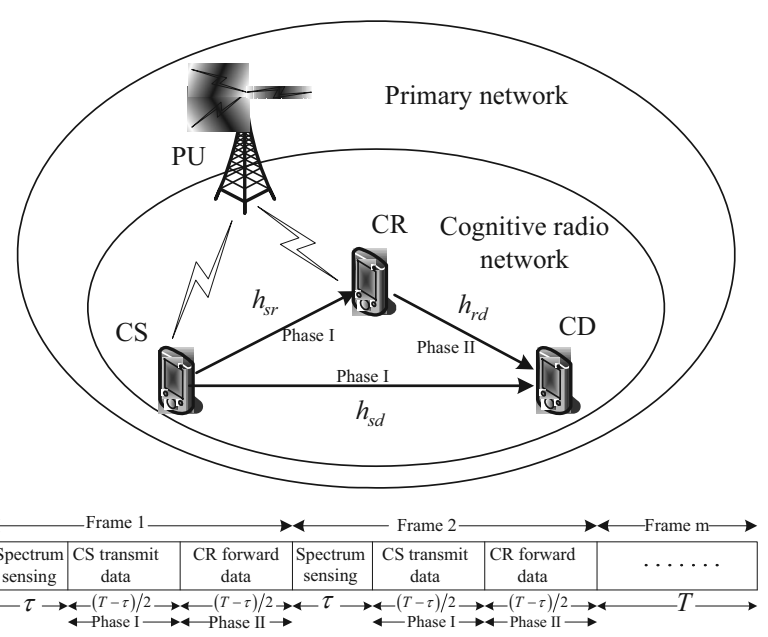

Figure 1. System model. pose that $\tau$ is the spectrum sensing duration and frame duration is $T$. So data transmission duration is $T-\tau$.

Assuming that channel state information is only known by the receiver but not by the transmitter, where $h_{s r}, h_{s d}$ and $h_{r d}$ denote the fading channel coefficients between CS and CR, CS and CD, $\mathrm{CR}$ and $\mathrm{CD}$ respectively, each transmission link between any two nodes is modeled as a Rayleigh fading process with variances $\delta_{s d}^{2}, \delta_{s r}^{2}$ and $\delta_{r d}^{2}$.

\section{Throughput and Energy Efficiency Optimization}

This section investigates the optimization problem of throughput and energy efficiency based on cognitive relay network.

\subsection{Throughput Optimization}

Once cognitive user detected idle licensed spectrum hole, cognitive radio networks would begin data transmission. Data transmission uses orthogonal channels, either through time or frequency division. The whole process of data transmission can be divided into two phases: 1) cognitive source broadcasts data to cognitive relay and cognitive destination in phase I, and 2) cognitive relay forward data to cognitive destination in AF mode, while cognitive source keeps silent during phase II. Finally, cognitive destination combines received data through maximal-ratio combining.

If primary user is idle, the capacity of cognitive relay networks can be represented as $C_{0}$. When primary user is active, the capacity that cognitive relay networks can achieve is $C_{1}$. The overall throughput of cognitive relay networks can be written as

$$
\begin{aligned}
T h(\eta, \tau) & =\frac{T-\tau}{T}\left(C_{0}\left(1-P_{f}(\eta, \tau)\right) P\left(H_{0}\right)\right. \\
& \left.+C_{1}\left(1-P_{d}(\eta, \tau)\right) P\left(H_{1}\right)\right)
\end{aligned}
$$

where $\eta$ is detection threshold, $P\left(H_{0}\right)$ and $P\left(H_{1}\right)$ are the probability that primary user is inactive and active respectively, and meet $P\left(H_{0}\right)+$ $P\left(H_{1}\right)=1 . P_{f}(\eta, \tau)$ is the probability of false alarm, $P_{d}(\eta, \tau)$ is detection probability. The 
false alarm probability related to the target detection probability $\bar{P}_{d}$ is as follows

$$
P_{f}(\tau)=Q\left[Q^{-1}\left(\bar{P}_{d}\right) \sqrt{\left(2 \gamma_{p}+1\right)}+\gamma_{p} \sqrt{\tau f_{s}}\right]
$$

where $f_{s}$ denotes sampling frequency, $\gamma_{p}$ is the received SNR of primary user signal measured at the cognitive receiver of interest under the hypothesis $H_{1} . Q(x)$ is the complementary distribution function of standard Gaussian, i.e.

$$
Q(x)=\frac{1}{\sqrt{2 \pi}} \int_{x}^{+\infty} e^{-\frac{t^{2}}{2}} d t .
$$

The average throughput of the cognitive relay network can be maximized by using $\tau$ and $\eta$ as the optimization variables subject to enough protection given to the primary user, the throughput optimization problem can be written as follows:

$$
\begin{gathered}
\max _{\eta, \tau} T h(\eta, \tau) \\
\text { s.t. } \quad P_{d}(\eta, \tau) \geq \bar{P}_{d} \\
0<\tau<T
\end{gathered}
$$

In cognitive radio networks, primary user should be protected sufficiently, the objective detection probability is satisfied with the constraint of $\bar{P}_{d} \geq 0.9$. Supposing that spectrum usage probability $P\left(H_{1}\right)$ is low, the throughput of cognitive relay networks can be approximately written as follows:

$$
T h(\eta, \tau) \approx \frac{T-\tau}{T} C_{0}\left(1-P_{f}(\eta, \tau)\right) P\left(H_{0}\right)
$$

$C_{0}$ is as follows [9]

$$
C_{0}=\frac{1}{2} \log _{2}\left(1+\frac{P_{s}\left|h_{s d}\right|^{2}}{\sigma_{n}^{2}}+\frac{\beta^{2} P_{s}\left|h_{s r}\right|^{2}\left|h_{r d}\right|^{2}}{\left(1+\beta^{2}\left|h_{r d}\right|^{2}\right) \sigma_{n}^{2}}\right)
$$

where $\beta=\sqrt{\frac{P_{r}}{P_{s}\left|h_{s, r}\right|^{2}+\sigma_{n}^{2}}}$ is the amplifier gain [9], $P_{s}=P_{r}$ is transmit power of cognitive source and cognitive relay respectively under the constraint of $P_{s}+P_{r}=P, P$ is allowable total power, $\sigma_{n}^{2}$ is variance of Gaussian noise. For a given objective detection probability $\bar{P}_{d}$, differentiating $T h(\tau)$ with respect to $\tau$ yields

$$
\begin{aligned}
\nabla T h(\tau) & =\frac{d T h}{d \tau}=-\frac{1}{T} P\left(H_{0}\right)\left(1-P_{f}(\tau)\right) C_{0} \\
& -\frac{T-\tau}{T} P\left(H_{0}\right) C_{0} \nabla P_{f}(\tau) .
\end{aligned}
$$

For the given objective detection probability $\bar{P}_{d}$, the false alarm probability can be written as follows:

$$
\begin{aligned}
P_{f}(\tau) & =Q\left[Q^{-1}\left(\bar{P}_{d}\right) \sqrt{\left(2 \gamma_{p}+1\right)}+\gamma_{p} \sqrt{\tau f_{s}}\right] \\
& =\frac{1}{\sqrt{2 \pi}} \int_{Q^{-1}\left(\bar{P}_{d}\right) \sqrt{\left(2 \gamma_{p}+1\right)}+\gamma_{p} \sqrt{\tau f_{s}}}^{\infty} e^{-\frac{t^{2}}{2}} d t .
\end{aligned}
$$

Differentiating $P_{f}(\tau)$ with respect to $\tau$ yields

$$
\begin{aligned}
& \nabla P_{f}(\tau)=\frac{d P_{f}}{d \tau}=-\frac{1}{\sqrt{2 \pi}} \frac{\gamma_{p} \sqrt{f_{s}}}{2 \sqrt{\tau}} \\
& \cdot \exp \left(-\frac{\left(Q^{-1}\left(\bar{P}_{d}\right) \sqrt{\left(2 \gamma_{p}+1\right)}+\gamma_{p} \sqrt{\tau f_{s}}\right)^{2}}{2}\right) .
\end{aligned}
$$

From formulas (7) and (8), we have $\lim _{\tau \rightarrow 0} P_{f}(\tau)=$ 1 and $\lim _{\tau \rightarrow 0} \nabla P_{f}(\tau)=-\infty$. When $\tau \rightarrow T$, $Q^{-1}\left(\bar{P}_{d}\right) \sqrt{\left(2 \gamma_{p}+1\right)}+\gamma_{p} \sqrt{\tau f_{s}}$ can achieve the maximum, and we have $\lim _{\tau \rightarrow T} \nabla P_{f}(\tau)=0$ and $\lim _{\tau \rightarrow T} P_{f}(\tau)=0$. Consequently, we can draw a conclusion as follows:

$$
\lim _{\tau \rightarrow 0} \nabla T h(\tau)=\infty \text { and } \lim _{\tau \rightarrow T} \nabla T h(\tau)<0
$$

From formula (9), it is easy to see that there exists sensing time $\tau$ meet that $\nabla T h(\tau)=$ $\frac{d T h(\tau)}{d \tau}=0$ over the period $0 \leq \tau \leq T$. Differentiating $\nabla T h(\tau)$ with respect to $\tau$ yields

$$
\begin{aligned}
\nabla^{2} T h(\tau) & =\frac{d^{2} T h}{d \tau^{2}} \\
& =\frac{2}{T} P\left(H_{0}\right) \nabla P_{f}(\tau) R \\
& -\frac{T-\tau}{T} P\left(H_{0}\right) R \nabla^{2} P_{f}(\tau)
\end{aligned}
$$

where the second derivative of $P_{f}(\tau)$ is given as 
follows:

$$
\begin{aligned}
& \nabla^{2} P_{f}(\tau)=\frac{d^{2} P_{f}}{d \tau^{2}}=\frac{1}{\sqrt{2 \pi}} \frac{\gamma_{p} \sqrt{f_{s}}}{4 \tau \sqrt{\tau}} \\
& \cdot \exp \left(-\frac{\left(Q^{-1}\left(\bar{P}_{d}\right) \sqrt{\left(2 \gamma_{p}+1\right)}+\gamma_{p} \sqrt{\left.\tau f_{s}\right)^{2}}\right.}{2}\right) \\
& -\frac{1}{\sqrt{2 \pi}} \frac{\gamma_{p} \sqrt{f_{s}}}{2 \sqrt{\tau}} \\
& \cdot \exp \left(-\frac{\left(Q^{-1}\left(\bar{P}_{d}\right) \sqrt{\left(2 \gamma_{p}+1\right)}+\gamma_{p} \sqrt{\tau f_{s}}\right)^{2}}{2}\right) \\
& \times\left(Q^{-1}\left(\bar{P}_{d}\right) \sqrt{\left(2 \gamma_{p}+1\right)}+\gamma_{p} \sqrt{\tau f_{s}}\right) \frac{\gamma_{p} \sqrt{f_{s}}}{2 \sqrt{\tau}} .
\end{aligned}
$$

From formula (11), we know that $\lim _{\tau \rightarrow 0} \nabla^{2} P_{f}(\tau)$ $=\infty$ and $\lim _{\tau \rightarrow T} \nabla^{2} P_{f}(\tau)=0$. The second derivative of throughput with respect to $\tau$ can be obtained as follows:

$$
\lim _{\tau \rightarrow 0} \nabla^{2} T h(\tau)=-\infty, \lim _{\tau \rightarrow T} \nabla^{2} T h(\tau)=0 .
$$

From formula (12), we can find that $\nabla^{2} T h(\tau)=$ $\frac{d^{2} T h}{d \tau^{2}}<0$ for $\tau \in(0, T)$. Therefore, we can draw a conclusion that there exists optimal sensing time $\tau_{\text {opt }}$ to maximize the throughput of $\operatorname{cog}$ nitive relay networks.

Let $\gamma=\frac{0.5 P}{\sigma_{n}^{2}}, A=\left|h_{s d}\right|^{2}, B=\left|h_{s r}\right|^{2}$ and $C=\left|h_{r d}\right|^{2}, A, B$ and $C$ are greater than 0 . The formula (4) is given by

$$
\begin{aligned}
& T h(\gamma, \eta, \tau)=\frac{T-\tau}{2 T}\left(1-P_{f}(\eta, \tau)\right) P\left(H_{0}\right) \\
& \times \log _{2}\left(1+A \gamma+\frac{B C \gamma^{2}}{(B \gamma+C \gamma+1)}\right) .
\end{aligned}
$$

For given sensing time $\tau$ and objective detection probability $\bar{P}_{d}$. Differentiating $\nabla T h(\gamma)$ with respect to $\gamma$ yields

$$
\begin{aligned}
& \nabla T h(\gamma)=\frac{d T h}{d \gamma}=\frac{T-\tau}{2 T} P\left(H_{0}\right)\left(1-P_{f}(\eta, \tau)\right) \\
& \times \frac{A(B \gamma+C \gamma+1)^{2}+B C \gamma(B \gamma+C \gamma+2)}{\ln 2\left[(B \gamma+C \gamma+1)(A \gamma+1)+B C \gamma^{2}\right](B \gamma+C \gamma+1)} .
\end{aligned}
$$

From formula (14), we know that $\nabla T h(\gamma)>$ 0 . Consequently, we can conclude that the throughput of cognitive radio networks is monotonically increasing for parameter $\gamma$.

\subsection{Energy Efficiency Optimization}

In this section, we investigate the energy efficiency of cognitive relay networks. The function of energy efficiency is defined as follows

$$
\zeta(\gamma, \tau)=\frac{T h(\gamma, \tau)}{E(\gamma, \tau)}
$$

where $\gamma=\frac{P}{2 \sigma_{n}^{2}}$ is signal to noise ratio, $E(\gamma, \tau)$ is the average energy consumption of cognitive relay networks. There exist three kinds of energy consumption, such as circuit power energy consumption, spectrum sensing energy consumption and data transmission energy consumption. The average energy consumed by cognitive relay networks is formulated as follows

$$
\begin{aligned}
E(\gamma, \tau) & =\tau P_{\tau}+P_{c} T \\
& +(T-\tau) \gamma \sigma_{n}^{2}\left[P\left(H_{0}\right)\left(1-P_{f}(\tau)\right)\right. \\
& \left.+P\left(H_{1}\right)\left(1-P_{d}(\tau)\right)\right]
\end{aligned}
$$

where $P_{\tau}$ is spectrum sensing power, $P_{c}$ denotes circuit power.

Signal to noise ratio $\gamma$ and sensing time $\tau$ can be optimized to improve the energy efficiency of cognitive relay network. The objective is to maximize the energy efficiency while satisfying sensing performance constraint, which can be formulated as

$$
\begin{aligned}
& \max _{\gamma, \tau} \zeta(\gamma, \tau)= \\
& \frac{(T-\tau) P\left(H_{0}\right)\left(1-P_{f}(\tau)\right)}{\tau P_{\tau}+P_{c} T+(T-\tau) \gamma \sigma_{n}^{2}\left[P\left(H_{0}\right)\left(1-P_{f}(\tau)\right)+P\left(H_{1}\right)\left(1-P_{d}(\tau)\right)\right]} \\
& \times \frac{1}{2 T} \log _{2}\left(1+\left|h_{s d}\right|^{2} \gamma+\frac{\gamma^{2}\left|h_{s r}\right|^{2}\left|h_{r d}\right|^{2}}{\left(\left|h_{s r}\right|^{2} \gamma+\left|h_{r d}\right|^{2} \gamma+1\right)}\right) \\
& \text { s.t. } \quad 0<\tau<T \\
& 0<\gamma<\infty \\
& P_{d}(\tau) \geq \bar{P}_{d} .
\end{aligned}
$$

Assuming that $\tau$ is constant and let

$$
\begin{aligned}
a & =\tau P_{\tau}+P_{c} T, \\
b & =(T-\tau) \sigma_{n}^{2}\left[P\left(H_{0}\right)\left(1-P_{f}(\tau)\right)\right. \\
& \left.+P\left(H_{1}\right)\left(1-P_{d}(\tau)\right)\right], \\
c & =\frac{1}{2 T}(T-\tau) P\left(H_{0}\right)\left(1-P_{f}(\tau)\right),
\end{aligned}
$$




$$
\begin{gathered}
d=\left|h_{s d}\right|^{2}, \\
e=\left|h_{s r}\right|^{2}, \\
f=\left|h_{r d}\right|^{2} .
\end{gathered}
$$

Obviously, we know that $a, b, c, d, e$ and $f$ are constant and greater than zero. The reduced form of energy efficiency can be rewritten as follows

$$
\zeta(\gamma)=\frac{c}{a+b \gamma} \log _{2}\left(1+d \gamma+\frac{\gamma^{2} e f}{(e \gamma+f \gamma+1)}\right) .
$$

Clearly, we have

$$
\lim _{\gamma \rightarrow 0} \zeta(\gamma)=0
$$

and

$$
\begin{aligned}
& \lim _{\gamma \rightarrow \infty} \zeta(\gamma) \\
& =\lim _{\gamma \rightarrow \infty} \frac{c \log _{2}\left(1+d \gamma+\frac{\gamma^{2} e f}{(e \gamma+f \gamma+1)}\right)}{(a+b \gamma)} \\
& =\lim _{\gamma \rightarrow \infty} \frac{c\left(d+\frac{2 f e \gamma}{e \gamma+f \gamma+1}-\frac{f e \gamma^{2}(e+f)}{(e \gamma+f \gamma+1)^{2}}\right)}{(\ln 2) b\left(1+d \gamma+\frac{\gamma^{2} e f}{(e \gamma+f \gamma+1)}\right)} \\
& =0 .
\end{aligned}
$$

From equation (20) and (21), we know that $\zeta(\gamma)$ is a continuous positive function, and there exists certain value for $\gamma(0<\gamma<\infty)$ to meet that

$$
\frac{\partial \zeta(\gamma)}{\partial \gamma} \mid \bar{\gamma}=0
$$

For a given objective detection probability $\bar{P}_{d}$, let $A=P\left(H_{0}\right) \gamma \sigma_{n}^{2}, B=P\left(H_{1}\right)\left(1-\bar{P}_{d}\right) \gamma \sigma_{n}^{2}$ and $C=P_{c} T$. Assuming that $\gamma$ is constant, formula (17) can be written as follows

$$
T \zeta(\tau)=\frac{(T-\tau) P\left(H_{0}\right)\left(1-P_{f}(\tau)\right) C_{0}}{\tau P_{\tau}+C+(T-\tau)\left(A\left(1-P_{f}(\tau)\right)+B\right)} .
$$

Let $D=\frac{P\left(H_{0}\right) C_{0}}{T}$, differentiating $\zeta(\tau)$ with respect to $\tau$ yields

$$
\begin{aligned}
& \zeta(\tau)=\frac{\partial \zeta}{\partial \tau} \\
& =\frac{\partial\left(D(T-\tau)\left(1-P_{f}(\tau)\right)\right)}{\partial\left(\tau P_{\tau}+C+(T-\tau)\left(A\left(1-P_{f}(\tau)\right)+B\right)\right)} \\
& =\frac{\left[-D\left(\left(1-P_{f}(\tau)\right)+(T-\tau) \nabla P_{f}(\tau)\right)\right]}{\left[\tau P_{\tau}+C+(T-\tau)\left(A\left(1-P_{f}(\tau)\right)+B\right)\right]^{2}} \\
& \cdot \frac{\left[\tau P_{\tau}+C+(T-\tau)\left(A\left(1-P_{f}(\tau)\right)+B\right)\right]}{\left[\tau P_{\tau}+C+(T-\tau)\left(A\left(1-P_{f}(\tau)\right)+B\right)\right]^{2}} \\
& -\frac{D(T-\tau)\left(1-P_{f}(\tau)\right)}{\left[\tau P_{\tau}+C+(T-\tau)\left(A\left(1-P_{f}(\tau)\right)+B\right)\right]^{2}} \\
& \cdot \frac{\left[P_{\tau}-A\left(1-P_{f}(\tau)\right)-B-(T-\tau) A \nabla P_{f}(\tau)\right]}{\left[\tau P_{\tau}+C+(T-\tau)\left(A\left(1-P_{f}(\tau)\right)+B\right)\right]^{2}} .
\end{aligned}
$$

When $\tau \rightarrow 0$, we know that $\lim _{\tau \rightarrow 0} P_{f}(\tau)=1$ and $\lim _{\tau \rightarrow 0} \nabla P_{f}(\tau)=-\infty$. Since $A, B, C$ and $D$ are constant, we can conclude that

$$
\begin{aligned}
\lim _{\tau \rightarrow 0} \nabla \zeta(\tau) & =\lim _{\tau \rightarrow 0} \frac{\partial \zeta}{\partial \tau} \\
& =\frac{-D T(C+B T)(-\infty)}{(C+B T)^{2}} \\
& =\frac{D T \infty}{(C+B T)}>0 .
\end{aligned}
$$

When $\tau \rightarrow T$, we know that $\lim _{\tau \rightarrow T} P_{f}(\tau)=0$ and $\lim _{\tau \rightarrow T} \nabla P_{f}(\tau)=0$, we can achieve that

$$
\begin{aligned}
\lim _{\tau \rightarrow T} \nabla \zeta(\tau) & =\lim _{\tau \rightarrow T} \frac{\partial \zeta}{\partial \tau} \\
& =\frac{-D\left(T P_{\tau}+C\right)}{\left(T P_{\tau}+C\right)^{2}} \\
& =-\frac{D}{\left(T P_{\tau}+C\right)}<0
\end{aligned}
$$

From formula (25) and (26), we can conclude that there is sensing time meet that $\nabla \zeta(\tau)=0$ over the period $0 \leq \tau \leq T$. The optimal sensing time is obtained when the equality constraint in (17) is satisfied, which can maximize the energy efficiency of cognitive radio networks.

\section{Numerical and Simulation Results}

In this section, extensive simulations are resorted to investigate the optimization performance of energy efficiency and throughput in 
cognitive relay networks. In the following simulations, it is assumed that the channels between PU and secondary users, and between cognitive source and cognitive relay, and between cognitive source and cognitive destination, and between cognitive relay and cognitive destination experience Rayleigh fading. The modulated signal bandwidth of primary users is $6 \mathrm{MHz}$. Setting $f_{s}=6 \mathrm{MHz}, T=20 \mathrm{~ms}$, $P\left(H_{0}\right)=0.8, P_{c}=200 \mathrm{~mW}, P_{\tau}=100 \mathrm{~mW}$ and $\sigma_{n}^{2}=1$ (normalized noise variance). The target detection probability for cognitive user is set to $\bar{P}_{d}=0.95$ in order to provide PU with sufficient protection. In cognitive relay networks, $\delta_{s d}^{2}, \delta_{s r}^{2}$ and $\delta_{r d}^{2}$ are set to 1,5 and 5 . Cognitive source and cognitive relay are allocated to equal power, and the system signal to noise ratio is $S N R=\frac{P}{\sigma_{n}^{2}}$.

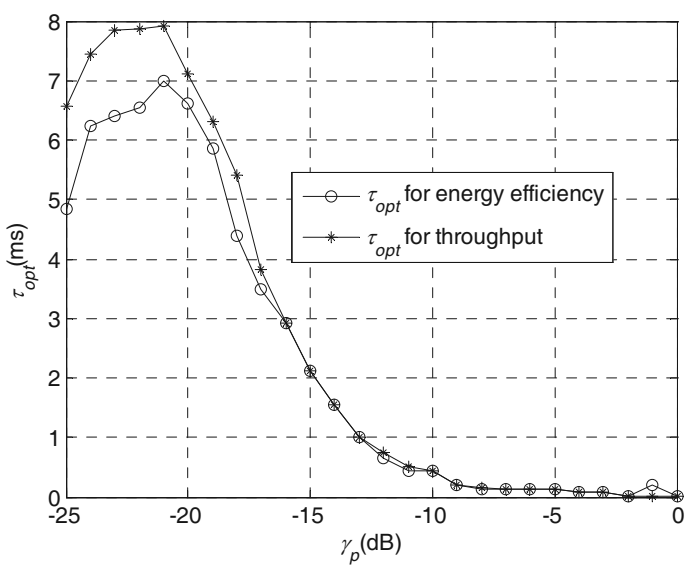

Figure 2. The optimal sensing time for throughput and energy efficiency maximization.

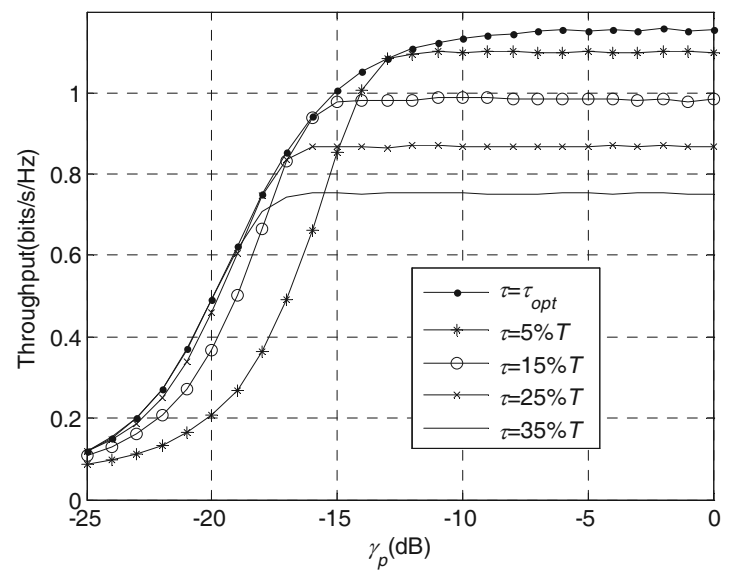

Figure 3. The throughput for different sensing time values.

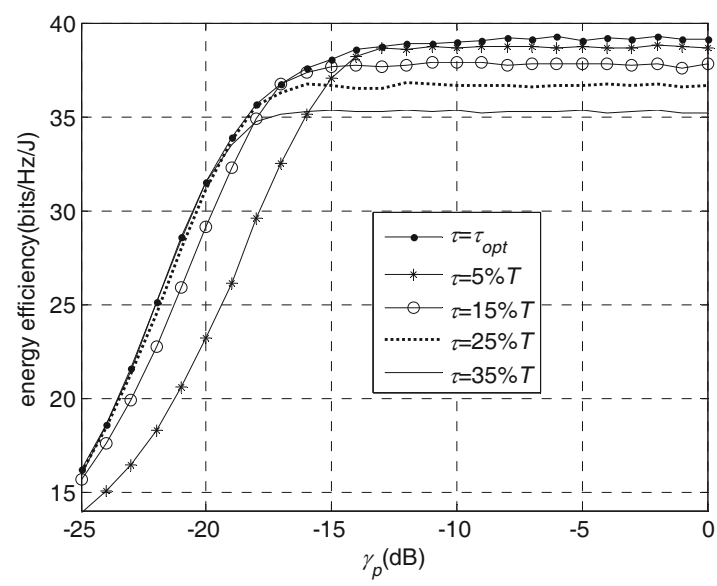

Figure 4. The energy efficiency for different sensing time values.

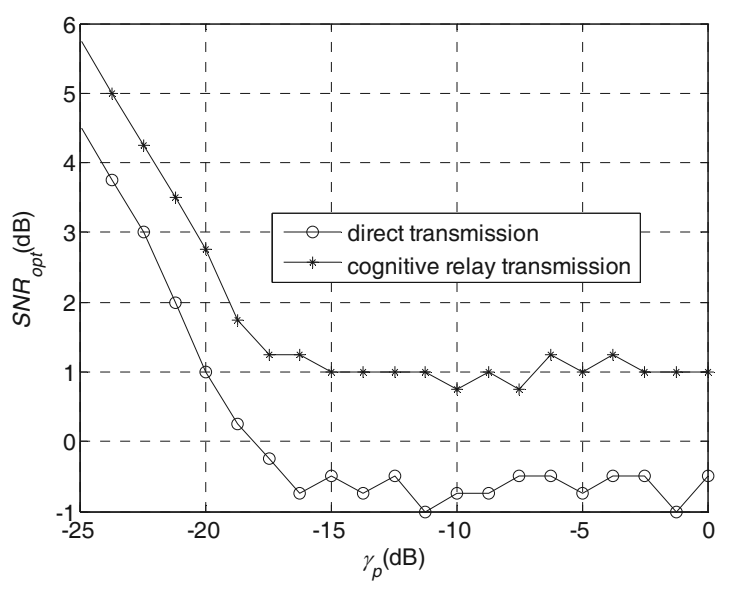

Figure 5. Optimal SNR for energy efficiency maximized versus $\gamma_{p}$.

Figure 2 illustrates that there exists the optimal sensing time $\tau_{\text {opt }}$ to make both the throughput and the energy efficiency of cognitive relay networks maximized under different $\gamma_{p}$. The optimal sensing time $\tau_{\text {opt }}$ of energy efficiency maximization is smaller than that of throughput maximization.

When $S N R=5 \mathrm{~dB}$, Figure 3 compares the throughput for sensing time $\tau=\tau_{\text {opt }}$ with other sensing time values, such as $\tau=5 \% T, 15 \% T$, $25 \% T$ and $35 \% T$. It is obvious that the throughput for optimal sensing time is the best. Similarly, it is easy to see from Figure 4 that the energy efficiency for $\tau=\tau_{\text {opt }}$ is the best.

Figure 5 illustrates the optimal $S N R$ for energy efficiency maximized under both direct transmission and cognitive relay transmission. We 
can know that there exists optimal SNR to maximize the energy efficiency of cognitive radio networks. Obviously, the optimal $S N R$ for cognitive relay transmission is smaller than that of direct transmission under different $\gamma_{p}$.

In Figure 6, we show the throughput of cognitive relay transmission versus sensing time under different $S N R$. One can see that there is optimal sensing time to maximize the throughput of cognitive radio networks.

Figure 7 depicts the energy efficiency of cognitive radio networks versus $S N R$ under different sensing time values. Note that there exists optimal $S N R$ to maximize the energy efficiency of cognitive radio networks. Furthermore, the energy of cognitive relay transmission is superior to that of direct transmission.

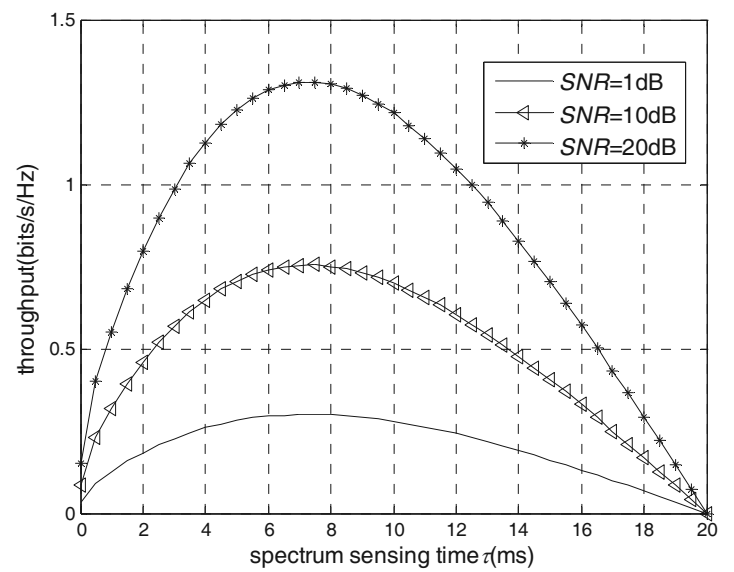

Figure 6. Throughput of cognitive relay transmission versus sensing time.

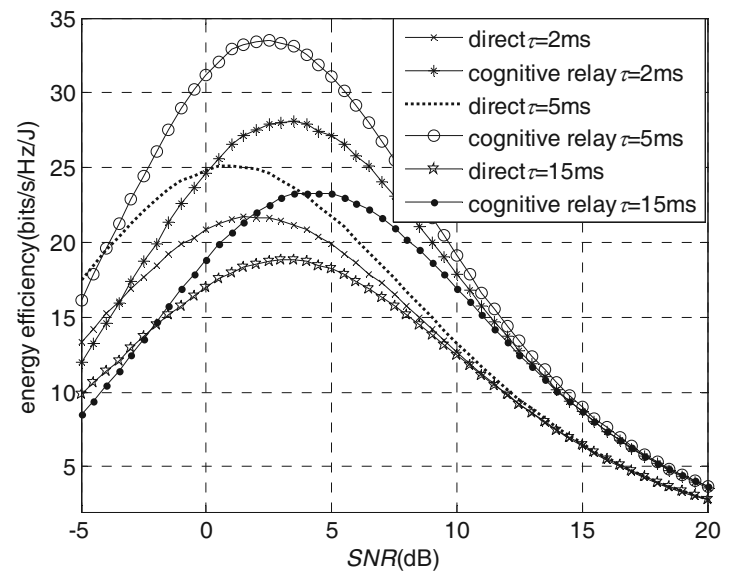

Figure 7. Energy efficiency of cognitive radio networks versus $S N R$.

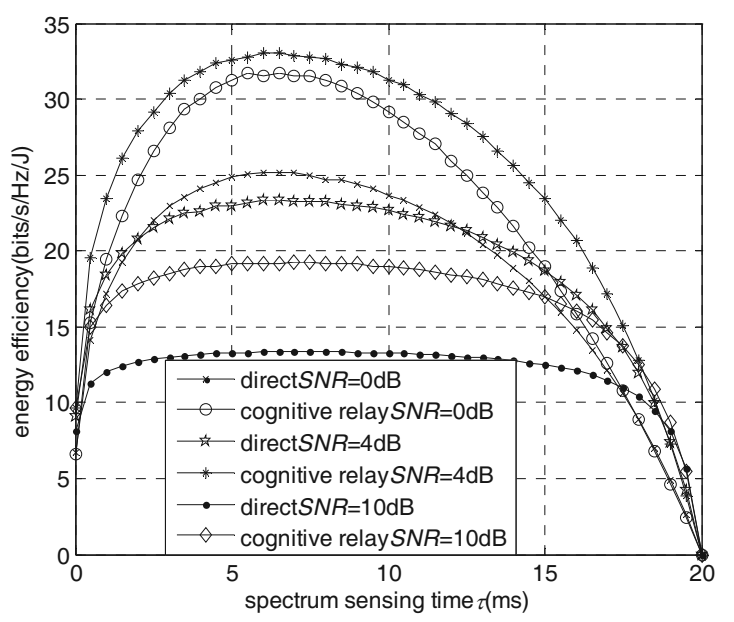

Figure 8. Energy efficiency of cognitive radio networks versus sensing time.

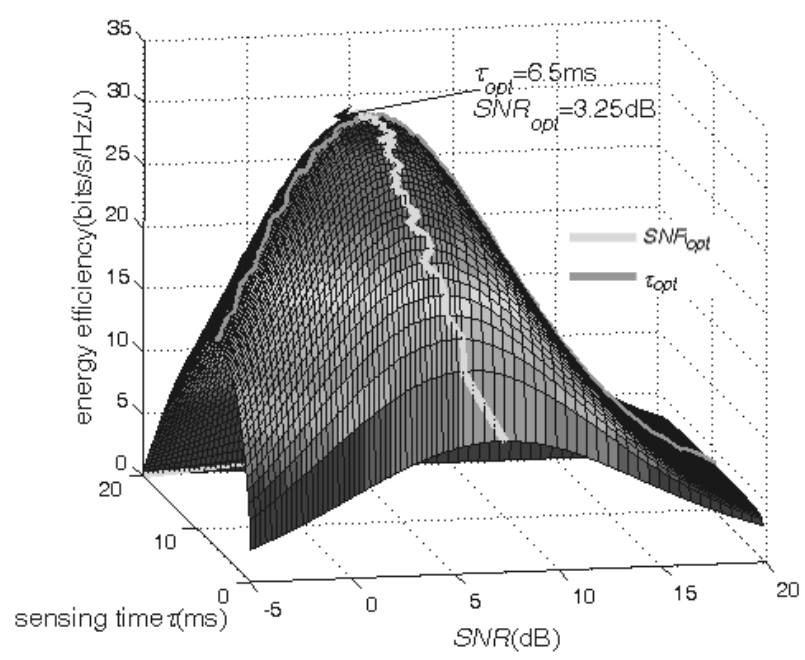

Figure 9. Energy efficiency of cognitive relay transmission versus sensing time and $S N R$.

Figure 8 shows that there is optimal sensing time to maximize the energy efficiency of cognitive radio networks under different $S N R$. When $S N R=4 \mathrm{~dB}$, the energy efficiency of both cognitive relay transmission scheme and direct transmission scheme outperforms that of other SNR.

Figure 9 illustrates three-dimensional graph of energy efficiency. It is obvious that there exists optimal sensing time and optimal $S N R$ to make the energy efficiency of cognitive relay networks maximized. When $\tau_{\text {opt }}=6.5 \mathrm{~ms}$, $S N R_{\text {opt }}=3.25 \mathrm{~dB}$, we can achieve the maximization of energy efficiency $\zeta_{\max }=34.3 \mathrm{bits} / \mathrm{s} / \mathrm{Hz} / \mathrm{J}$. 


\section{Conclusion}

In this paper, we have presented throughput and energy efficiency maximization based on cognitive relay network. Our idea proves that cognitive radio network can significantly improve both spectral efficiency and energy efficiency by optimally designing spectrum sensing duration and SNR. The energy efficiency of cognitive relay transmission scheme is superior to that of non-relay transmission scheme.

\section{Acknowledgment}

This work was supported by the Foundation of Yunnan Province under grant No. 2011FB035 and School training fund.

\section{References}

[1] C. Cormio, K. R. Chowdhury, A survery on mac protocols for cognitive radio networks. Ad Hoc Networks, 7 (2007), 1315-1329.

[2] Y. C. Liang, Y. Zeng, E. C. Y. Peh, A. T. Hoang, Sensing throughput tradeoff for cognitive radio networks. IEEE Trans. Wireless Communications, 7(4) (2008), 1326-1337.

[3] P. P. CHEN, Q. Y. ZHANG, Joint temporal and spatial sensing based cooperative cognitive networks. IEEE Commun. Lett., 15(5) (2011), 530-532.

[4] A. O. ERCAN, M. O. SunAY, Energy sensing strategy optimization for opportunistic spectrum access. IEEE Commun. Lett., 16(6) (2012), 828-830.

[5] P. YiYANG, L. YING-CHANG, Energy-Efficient Design of Sequential Channel Sensing in Cognitive Radio Networks: Optimal Sensing Strategy, Power Allocation, and Sensing Order. Selected Areas in Communications, IEEE Journal, 29(8) (2001), 1648-1659.

[6] L. LI, X. ZHOU, H. Xu, Y. LI, D. WANG, A. SoONG, Energy-efficient transmission in cognitive radio networks. IEEE Trans. Broadcast., 57(3) (2011), 718 720 .

[7] Y. WU, D. H. K. TSANG, Energy-efficient spectrum and transmission for cognitive radio system. IEEE Commun. Lett., 15(5) (2011), 545-547.

[8] L. LiYing, Z. XiangweI, Energy-Efficient Transmission in Cognitive Radio Networks. Consumer Communications and Networking Conference (CCNC), 7th IEEE, (2010).
[9] J. N. Laneman, D. N. C. Tse, G. W. Wornell, Cooperative Diversity in Wireless Networks: Efficient Protocols and Outage Behavior. Information Theory, IEEE Transactions, 50 (2004), 3062-3080.

[10] C. Huang, R. Zhang, S. CuI, Throughput maximization for the gaussian relay channel with energy harvesting constraints. IEEE J. Sel. Areas Commun., 31(8) (2013), 1469-1479.

[11] S. PARK, H. KIM, D. Hong, Cognitive radio networks with energy harvesting. IEEE Trans. Wireless Commun., 12(3) (2013), 1386-1397.

[12] S. PARK, D. Hong, Achievable throughput of energy harvesting cognitive radio networks. IEEE Transactions on Wireless Communications, 13(2) (2014), 1010-1022.

Received: May, 2014

Revised: July, 2014

Accepted: July, 2014

Contact addresses:

Yaolian Song

Faculty of Information Engineering and Automation Kunming University of Science and Technology

Kunming, 650504

e-mail: sy1719@163.com

Fan Zhang

Faculty of Information Engineering and Automation Kunming University of Science and Technology

Kunming, 650504

China

Shao Yubin

Faculty of Information Engineering and Automation Kunming University of Science and Technology Kunming, 650504

China

YAOLIAN SONG graduated from Nanjing University of Posts and Telecommunications (NUPT), Ph.D., Wireless Communication Major at Kunming University of Science and Technology (KMUST), Information Engineering and Communication Department. She is concentrating on the area of wireless communication and cognitive radio.

FAN ZHANG is a Master graduate student of Professional Electronic and Communication System at Kunming University of Science and Technology, School of the Faculty of Information Engineering and Automation.

SHAO YUBIN graduated from the University of Electronic Science and Technology of China (UESTC) in the field of Communication Engineering in 1991. He is working now as a Professor at Kunming University of Science and Technology (KMUST), concentrating on the area of wireless communication technology, especially on the anti-interference and MIMO communications, as a research associate at the University of New South Wales(UNSW). 